\title{
Endobronchial Valve Used as Salvage Therapy in a Mechanically Ventilated Patient with Intractable Life-Threatening Haemoptysis
}

\author{
Usha Lalla Brian W. Allwood Soumitra Sinha Roy Elvis M. Irusen \\ Coenraad F.N. Koegelenberg \\ Division of Pulmonology, Department of Medicine, Stellenbosch University and Tygerberg Academic Hospital, \\ Cape Town, South Africa
}

\section{For editorial comment see p. 398}

\section{Established Facts}

- Treatment options for intractable life-threatening haemoptysis in mechanically ventilated patients with structural lung disease who are deemed unfit for surgery are limited.

\section{Novel Insights}

- Endobronchial valves can potentially be used to control ongoing life-threatening haemoptysis and liberate mechanically ventilated patients from invasive ventilation.

\section{Keywords}

Endobronchial valve · Haemoptysis

\begin{abstract}
Treatment options for intractable life-threatening haemoptysis in mechanically ventilated patients with structural lung disease who do not respond to bronchial artery embolisation (BAE) and who are deemed unfit for surgery are limited. A 26-year-old HIV-positive male with a poorly preserved $\mathrm{CD}_{4}$ count and active pulmonary tuberculosis was intubated and mechanically ventilated for persistent life-threatening haemoptysis. Two attempts at BAE failed, and life-threatening
\end{abstract}

haemoptysis recurred daily for 14 days despite antituberculous therapy. He was deemed unfit for surgery during that period. We proceeded to identify the source of bleeding endoscopically and implanted an endobronchial valve in the left upper lobe bronchus. Following the collapse of the affected lobe, haemoptysis ceased and the patient was promptly liberated from mechanical ventilation. He remained haemoptysis free for the duration of his hospitalisation. Endobronchial valves, therefore, may be a viable option in patients mechanically ventilated with persistent lifethreatening haemoptysis in whom all other conventional measures fail and who are considered unfit for surgery.

(c) 2017 S. Karger AG, Basel

\section{KARGER}

(C) 2017 S. Karger AG, Basel 


\section{Introduction}

Active tuberculosis and its sequelae, including bronchiectasis and aspergillomata, are still the most common causes of haemoptysis in the developing world, where life-threatening haemoptysis remains a common and not infrequently fatal medical emergency $[1,2]$. Haemodynamic and ventilatory support, followed by bronchial artery embolisation (BAE), are the preferred initial treatment in most institutions [1]. Patient position with the bleeding side dependant and pulmonary isolation with either a double-lumen tube or selective bronchial intubation can be employed as temporary measures $[3,4]$. Endoscopic management is generally limited to procedures aiming at achieving tamponade and haemostasis [4]. Endoscopic electrocautery, argon plasma coagulation, cryotherapy and related procedures, which are frequently utilised to control haemoptysis secondary to lung cancer, generally have no role in the management of haemoptysis in the setting of tuberculosis or structural lung disease [5].

Recurrence of haemoptysis following BAE for lifethreatening haemoptysis is common and is associated with a high mortality [1]. Residual haemoptysis beyond the first week after embolisation, multiple blood transfusions and the presence of an aspergilloma are all associated with an increased risk of recurrence of life-threatening haemoptysis [1]. Conversely, the presence of active tuberculosis amenable to treatment lowers the risk [1]. Emergency surgery, usually aimed at resection of the affected lobe(s), is often the only recourse, provided pulmonary reserve and other comorbidities do not preclude this course of action [6].

We previously described the successful use of intrabronchial valves in a haemodynamically stable patient with bilateral aspergillomata which precluded surgery [7]. Other devices, particularly spigots, have also been used in similar cases to achieve tamponade $[8,9]$.

\section{Case Report}

A 26-year-old HIV-positive male with a $\mathrm{CD}_{4}$ count of 126 cells/ $\mathrm{mm}^{3}$ was transferred to our intensive care unit (ICU) following life-threatening haemoptysis. He was intubated and in hypovolaemic shock with a haemoglobin of $5.5 \mathrm{~g} / \mathrm{dL}$. No coagulopathy was present at the time of admission. The patient was diagnosed with pulmonary tuberculosis days prior to admission with a positive sputum Xpert MTB/RIF assay indicating that he was rifampicin sensitive (Cepheid Inc., Sunnyvale, CA, USA). His chest radiograph on admission revealed bronchopneumonic features with a clear cavity in the left upper lobe (LUL) (Fig. 1). Following haemodynamic stabilisation, involving numerous blood transfusions and

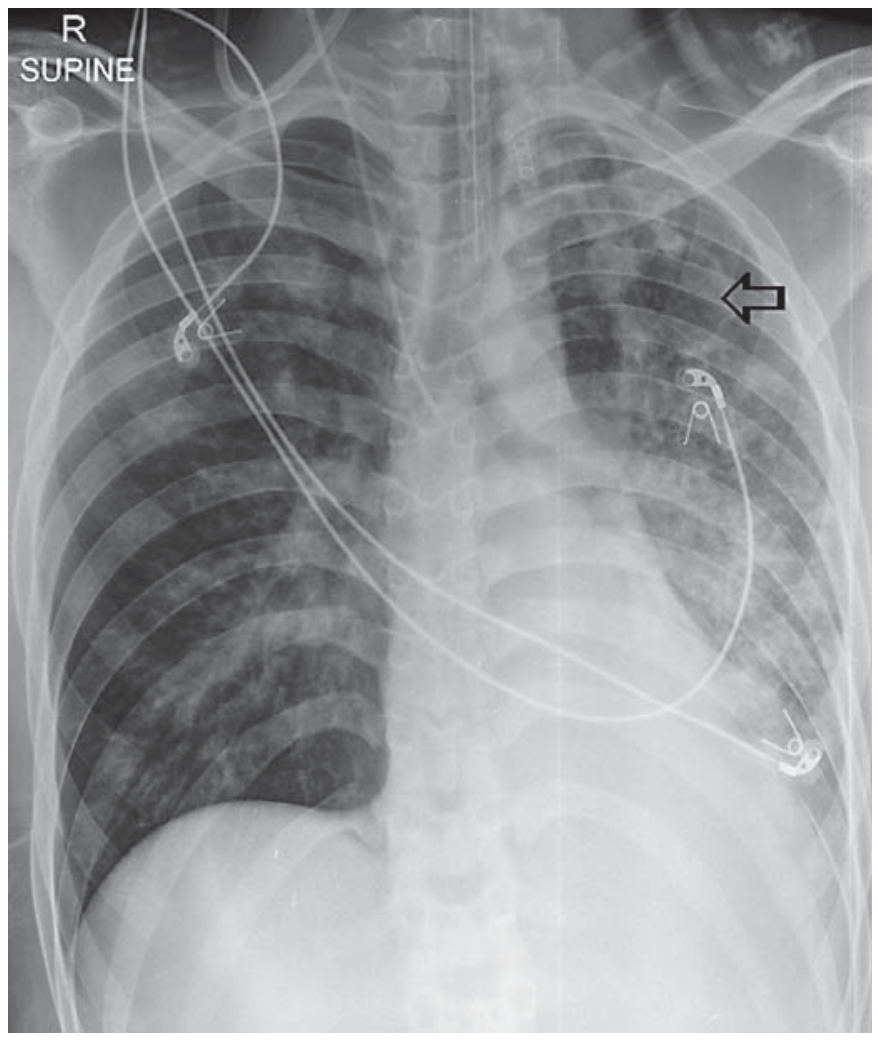

Fig. 1. The patient's chest radiograph taken on admission to the intensive care unit. Note the cavity in the left upper lobe (arrow).

vasopressor support, he underwent urgent bronchial artery angiography which was unable to localise a focus of bleeding.

He was initially selectively ventilated via the right main bronchus but later ventilated by means of a standard endotracheal intubation. Over the course of the next few days, the patient was nursed in the left lateral position and deeply sedated to prevent further haemoptysis. His left main bronchus was occluded on several occasions (Fig. 2), presumably secondary to blood clots. A computed tomography scan performed days after admission also confirmed that the left main bronchus was occluded by debris, resulting in complete atelectasis of the left lung, which unfortunately precluded assessment of fissural intactness on the left.

When he improved sufficiently from a ventilation and haemodynamic perspective, a trial of spontaneous breathing was attempted. This was promptly aborted due to the recurrence of massive haemoptysis and haemorrhagic shock, necessitating repeat blood transfusion and recommencement of vasopressors. After consultation with the cardiothoracic surgeons he was deemed a poor candidate for urgent surgical intervention in view of the significant morbidity and mortality associated with the presence of active tuberculosis, the need for mechanical ventilation and his immunocompromised state.

Subsequent attempts at permitting spontaneous respiration resulted in a similar outcome, and he ultimately required muscle 


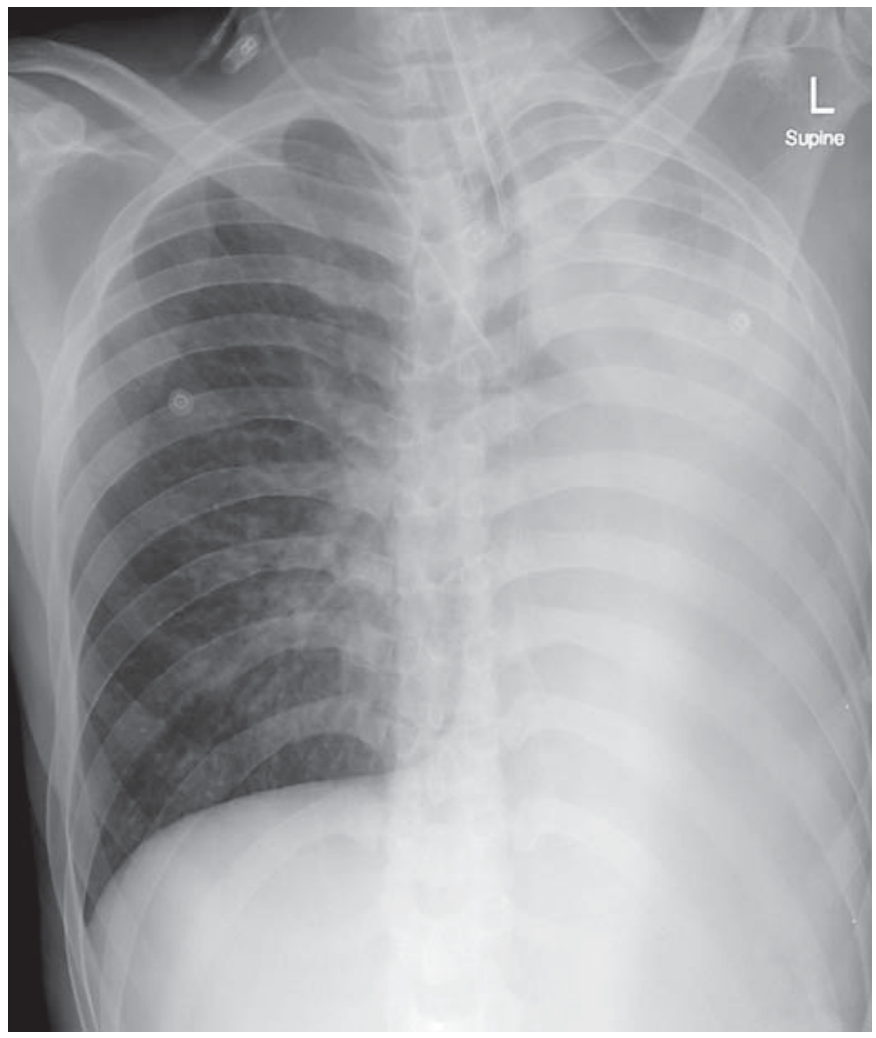

Fig. 2. One of several chest radiographs taken during admission showing complete collapse of the left lung.

paralysis to prevent further haemoptysis. The BAE was repeated, and an aberrant vessel was identified just below the level of the carina on the left. This was unfortunately a very small-calibre vessel with an acute take-off angle, and secure engagement with the catheter could not be achieved. A second, large-calibre vessel was identified on the right, supplying the same arterial bed on the left. This vessel was successfully embolised with polyvinyl alcohol particles. Unfortunately, he continued to expectorate large volumes of blood via the endotracheal tube, commencing shortly after the affected lung had re-expanded and spontaneous respiration had been resumed, making liberation from mechanical ventilation impossible.

After 14 days and having exhausted all available options to control life-threatening haemoptysis, we considered a minimally invasive approach to induce and maintain collapse of the LUL, given the success of the preceding spontaneous collapse of the affected lung in arresting haemoptysis.

After obtaining consent from the next of kin as well as our local hospital ethics committee, we proceeded to perform a flexible bronchoscopy (with a 3.2-mm working channel) via an 8.5 endotracheal tube (with rigid bronchoscopy available as a backup). The left main bronchus was completely occluded by blood clots, which were carefully suctioned. All lobar bronchi were occluded as well, and we proceeded to suction the left lower lobe (all segments), followed by the LUL proximal to the lingula, the lingula and finally

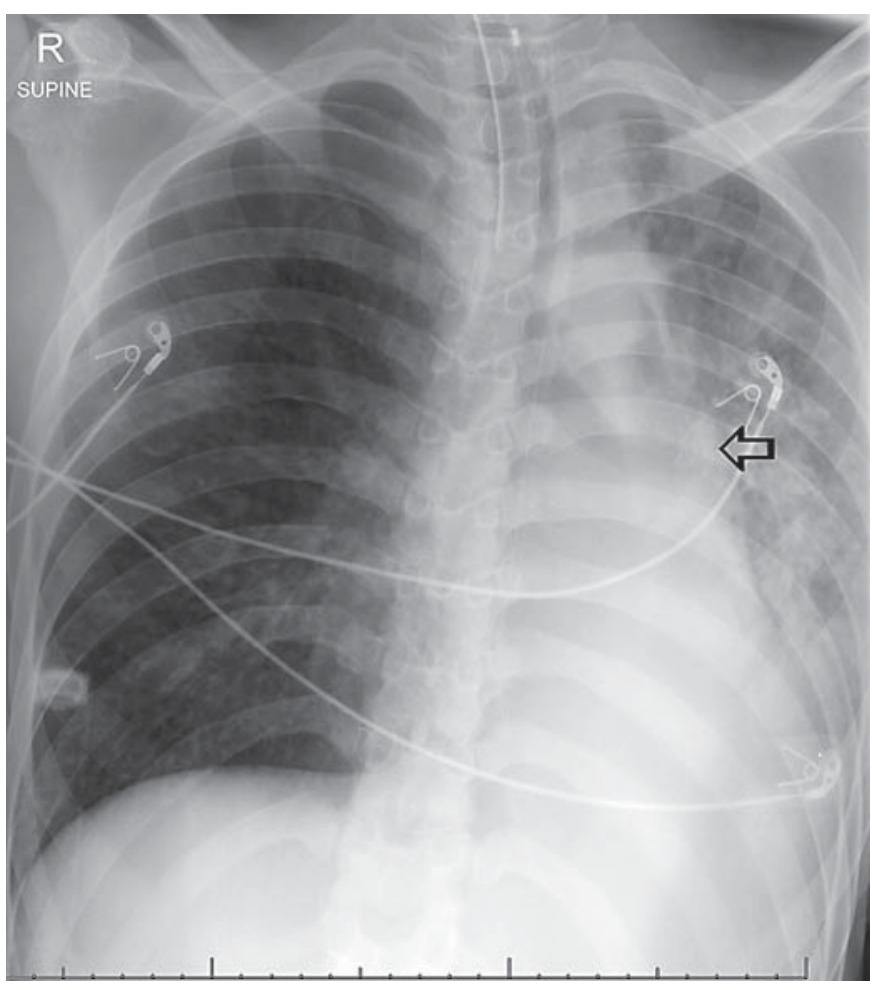

Fig. 3. The patient's chest radiograph hours after the implantation of the endobronchial valve. Note the deployed valve (arrow), partial left upper lobe collapse (including collapse of the cavity that was visible in Fig. 1) and volume reduction of the left lung.

the anterior and apicoposterior segment of the LUL. The apicoposterior segment of the LUL, as anticipated, was the source of active bleeding. A size 5.5 Zephyr endobronchial valve (PulmonX Inc., Redwood City, CA, USA) was implanted in the LUL bronchus distal to the lingula with no procedural complications. Evaluation for collateral ventilation (by means of a Chartis System) was not performed prior to deployment, as this would have prolonged the procedure and was considered too high a risk in this particular case. Moreover, the measurement of air flow in airways that contained ample clotted and fresh blood may very well have been inaccurate on that day.

The chest X-ray taken after the procedure confirmed adequate valve placement and deployment, no pneumothorax and complete atelectasis of the LUL distal to the lingula (Fig. 3). The patient was deeply sedated and mechanically ventilated for $48 \mathrm{~h}$ after the procedure and was subsequently extubated with no further haemoptysis. Following discharge from the ICU, he remained haemoptysis free during his hospitalisation. Anti-retroviral therapy was commenced 2 weeks after ICU discharge. During the course of the next 6 months he continued to remain haemoptysis free. No valve-related complications, including pneumonia, were observed. We opted to remove the valve after 6 months, given his excellent functional improvement and spontaneous partial re-expansion of the LUL. The procedure was uneventful. 


\section{Discussion}

Endobronchial valves were designed for endoscopic lung volume reduction by interrupting the entrance of air during inspiration and to permit exhalation of air and secretions [10]. In the ideal candidate (severe hyperinflation with no interlobar collateral ventilation) they have been proven to be effective [11]. They are also licensed, and often used, for prolonged air leaks (bronchopleural fistulae) [12]. However, these valves, to the best of our knowledge, have never been utilised as an emergency intervention for persistent life-threatening haemoptysis in a mechanically ventilated vasopressor-dependent patient with structural lung disease where all medical interventions failed and who was considered inoperable.

Rigid bronchoscopy is the norm in the acute management of acute life-threatening haemoptysis not responsive to BAE [13]. Treatment options in the setting of structural lung disease include endoscopic instillation of a fibrinogen-thrombin combination, various balloon tamponade devices (endobronchial balloon blockers) and even self-expanding airway stents $[2,14]$. Endobronchial airway blockade, in essence, may achieve acute control, usually as a bridge to definitive therapy in the form of pulmonary resection [2]. Blockade with a silicone spigot was described almost a decade ago [9]. Dutau et al. [9] described the endobronchial placement of a spigot placed via flexible bronchoscopy in order to prevent alveolar inundation preceding and during the time of BAE.

We previously described the implantation of an $\mathrm{IBV}^{\circledR}$ intrabronchial valve (Olympus Respiratory America) in a patient with recurrent haemoptysis secondary to bilateral upper lobe aspergillomata [7]. The patient remained haemoptysis free for 6 months and to date [unpubl. data] and experienced an unexplained and rather dramatic improvement in functional capacity and lung function [7]. We postulated at the time that segmental collapse and haemostasis resulted in a functional tamponade, comparable to that achieved with a silicone spigot. In the present case, lobar collapse of the cavity in the LUL may have significantly contributed to the medium and long-term success. Corbetta et al. [15] very recently described the application of endobronchial 1-way valves to induce lobar volume reduction as an adjunct to drug treatment in patients with mycobacterial infection who were considered poor surgical candidates. They hypothesised that the use of valves could also induce closure of cavities by collapsing the involved lobe, resulting in a mechanical anaerobic environment unfavourable to the survival of mycobacteria, a reduction in the movement of the pulmonary parenchyma to promote a favourable effect on tissue repair and possibly an enhanced systemic pharmacological drug effect by promoting drug penetration into a smaller affected area [15].

Pulmonary resection remains the most definitive intervention in appropriate cases, e.g., where a clear single source of bleeding can be identified in patients with structural lung disease with life-threatening haemoptysis. Our case suggests that lobar or segmental collapse may be seen at least as a bridge to surgery in cases not responsive or amenable to BAE. Only prospective multicentre studies would be able to assess the utility of endobronchial valves in this setting and, in particular, whether or not cavitation in this setting is a conditio sine qua non. The greatest challenge in this regard is the availability, cost and expertise required, as the largest burden of tuberculosis and its sequelae is seen in the developing world.

\section{Acknowledgements}

The authors are grateful to High Tech Medical (South Africa), who donated the endobronchial valve.

\section{Financial Disclosure and Conflicts of Interest}

The authors declare that they have no conflicts of interest.

\section{References}

Endobronchial Valve for Life-Threatening Haemoptysis
1 van den Heuvel MM, Els Z, Koegelenberg CF, Naidu KM, Bolliger CT, Diacon AH: Risk factors for recurrence of haemoptysis following bronchial artery embolisation for life-threatening haemoptysis. Int J Tuberc Lung Dis 2007;11:909-914.

2 Gross A, Diacon A, van den Heuvel M, Janse van Rensburg J, Harris D, Bolliger C: Management of life-threatening haemoptysis in an area of high tuberculosis incidence. Int $\mathrm{J} \mathrm{Tu}$ berc Lung Dis 2009; 13:875-880.
3 Jean-Baptiste E: Clinical assessment and management of massive hemoptysis. Crit Care Med 2000;28:1642-1647.

4 Anantham D, Jagadesan R, Tiew P: Clinical review: independent lung ventilation in critical care. Crit Care 2005;9:594-600.

5 Kvale P, Selecky P, Prakash U: Palliative care in lung cancer: ACCP evidence-based clinical practice guidelines (2nd edition). Chest 2007; 132(suppl 3):368S-403S. 
6 Cahill B, Ingbar D: Massive hemoptysis. Assessment and management. Clin Chest Med 1994;15:147-167.

7 Koegelenberg CFN, Bruwer JW, Bolliger CT: Endobronchial valves in the management of recurrent haemoptysis. Respiration 2014;87: 84-88.

8 Watanabe Y, Matsuo K, Tamaoki A, Komoto R, Hiraki S: Bronchial occlusion with endobronchial Watanabe spigot. J Bronchol 2003; 10:264-267.

9 Dutau H, Palot A, Haas A, Decamps I, Durieux O: Endobronchial embolization with a silicone spigot as a temporary treatment for massive hemoptysis: a new bronchoscopic approach of the disease. Respiration 2006;73: 830-832.
10 Koegelenberg CFN, Slebos D-J, Shah PL, et al: Time for the global rollout of endoscopic lung volume reduction. Respiration 2015;90:430440.

11 Valipour A, Slebos D, Herth F, et al: Endobronchial valve therapy in patients with homogeneous emphysema. Results from the IMPACT Study. Am J Respir Crit Care Med 2016;194:1073-1082.

12 Dooms CA, De Leyn PR, Yserbyt J, Decaluwe $\mathrm{H}$, Ninane V: Endobronchial valves for persistent postoperative pulmonary air leak: accurate monitoring and functional implications. Respiration 2012;84:329-333.
13 Sakr L, Dutau H: Massive hemoptysis: an update on the role of bronchoscopy in diagnosis and management. Respiration 2010;80:3858.

14 Freitag L, Tekolf E, Stamatis G, Montag M, Greschuchna D: Three years experience with a new balloon catheter for the management of haemoptysis. Eur Respir J 1994;7:2033-2037.

15 Corbetta L, Tofani A, Montinaro F, et al: Lobar collapse therapy using endobronchial valves as a new complementary approach to treat cavities in multidrug-resistant tuberculosis and difficult-to-treat tuberculosis: a case series. Respiration 2014;92:316-328. 